\title{
Improving the education and experience of foundation doctors in general surgery
}

\author{
Gareth Martel, Cathy Magee
}

NHS

\begin{abstract}
Reduced working hours and fragmentation of the surgical firm has resulted in a gradual change in FY1 duties. Locally, FY1 doctors were no longer routinely seeing surgical emergency admissions, while FY1s informally reported reduced confidence in dealing with surgical emergencies. The goal of this project was to assess the current training of FY1 doctors within the unit, and to attempt to improve this by increasing exposure to surgical emergencies.
\end{abstract}

Two months into their four month surgical rotation, FY1s completed an anonymous online survey that focused on their confidence in dealing with emergency surgical admissions. Working practice was then changed by the creation of a formal emergency foundation year one (FY1) rota, and the introduction of a baton bleep. The expectation was that all emergency admissions would be clerked by an FY1 doctor. The cohort were asked to repeat the survey after implementation of change.

Across all areas assessed, the confidence and experience of the junior doctors was improved. Initially $70 \%$ of FY1s felt exposure to emergency surgical cases was inadequate, falling to $0 \%$ after the intervention. This was associated with a rise in the average number of acute cases clerked by each FY1 per week from 1.2 to 4.0 . At baseline, only $30 \%$ of those surveyed felt that they were gaining the skills and experience necessary to prepare them for an FY2 job in general surgery, and after the intervention this increased to $100 \%$.

The increased pressures of service provision within reduced working hours does not necessitate the exclusion FY1 doctors from the assessment and management of surgical emergencies. We have demonstrated that preserving this exposure is a priority in the training of junior doctors, resulting in more experienced and confident medical staff.

\section{Problem}

New regulations regarding working practices for junior doctors have led to a significant reduction in hours spent in hospital. Within the general surgery department of Antrim Hospital, all foundation year one (FY1) doctors are working a maximum of 48 hours/week, including out of hours shifts. An unintentional effect of this is that the traditional team based surgical firm has been disrupted, with FY1s frequently changing teams to provide sufficient cover.

The reduction in working hours, with no compensatory increase in number of junior doctors available, has led to increased pressure to complete the daily tasks of running the surgical wards. Involvement of FY1s in acute surgical admissions has been sacrificed to ensure that routine tasks for existing ward patients are completed.(1) The assessment and management of acute referrals have been left to more senior members of the team, although management of emergencies should remain a key part of the training of junior doctors.(2) FY1s informally reported reduced confidence in dealing with acutely unwell surgical patients and dissatisfaction with their role, with the perception that they are less valued as part of the surgical team. This is reflected in the 2013 GMC training survey, with surgery scoring the lowest on foundation doctor satisfaction.(3)

\section{Background}

The pathway for acute surgical admissions traditionally involves initial review at senior house officer (SHO), often within the emergency department. This may be by either a foundation year two doctor (FY2), or by a core surgical trainee (CST). Subsequently the patient is admitted to the surgical unit, where a more senior assessment is carried out by the on-call registrar. Additionally, a thorough clerk-in would have been performed by the FY1, including implementation of initial management, and arranging relevant investigations. One of the main benefits of this structure was that it allowed the FY1 to gain exposure to surgical emergencies in a protected fashion. Through this they gained the clinical experience to support the transition to FY2 level the following year.

Reduction in working hours have led to degradation of this structure in many units, with the SHO (FY2-CT2) completing the traditional FY1 roles. The FY1 often has no involvement in the initial assessment and management of emergency admissions, and overall fewer training opportunities.(4)

\section{Baseline measurement}

An anonymised online survey was created to measure FY1s' exposure to surgical emergencies in their current post. Self-rated confidence in dealing with surgical emergencies was assessed, as was the degree to which they were being prepared for a role as an $\mathrm{SHO}$ in general surgery. 
On average the FY1s were assessing 1.2 acute surgical admissions per week, with a range from zero to three. None of the respondents at baseline felt that they were gaining adequate exposure to surgical emergencies.

With regards to management of acute surgical conditions, $60 \%$ $(n=6)$ of respondents felt confident in choosing basic investigations and instigating initial management, but none felt confident in their diagnostic skills. When specifically asked if they felt that they were being prepared for an SHO job in general surgery, only $30 \%(n=3)$ felt that the current training was adequate.

\section{Design}

A meeting with FY1s was arranged to establish potential changes to daily working patterns that would increase FY1 involvement in acute admissions. The existing rota allocated some FY1s to a flexible "floating" position, rather than being allocated to a specific team. These doctors provided cover at ward level on an ad hoc basis for teams with higher than normal workload, or for teams with no FY1 due to scheduled days off, night shifts or leave.

It was agreed that one "floating" doctor could be released without detriment to service provision and allocated to the emergency receiving team. An extra pager was obtained and designated as the on call baton bleep. This bleep was passed from the daytime emergency FY1 to the FY1 on the evening shift, and then to the FY1 on night shift who then returned it to the daytime FY1. In this manner, an FY1 responsible for acute admissions was always contactable.

A new policy was agreed whereby all acute surgical admissions were reviewed by the emergency FY1 and a full clerk-in completed. FY1s were encouraged to make the most of this experience, discussing cases they saw with more senior members of the team and taking the opportunity to complete work based assessments.

\section{Strategy}

PDSA cycle 1

An initial meeting was organised to establish what the barriers to seeing acute cases were within the current working pattern. The FY1s did not feel that there was an expectation or need for them to see acute admissions, and felt that their role was to complete ward based tasks as requested by nursing staff or the surgical team. This belief was challenged and the benefits of involvement in emergency care highlighted.

It was agreed that FY1s would endeavour to see every emergency admission to the unit. It was felt that the best way to achieve this would be that the FY1 doctor allocated to the on-call consultant would clerk all admissions during the day, evening admissions would be reviewed by the FY1 working the late shift, and the FY1 on night shift would clerk all the admissions overnight. In practice, while the doctors involved expressed positive feedback from the increased emergency exposure, we found that the FY1 doctor during the day was the most busy of all the junior doctors and therefore struggled with the extra workload of admitting emergency patients.

\section{PDSA cycle 2}

The system was adapted to involve one of the FY1 doctors allocated to "float". They became the emergency FY1, and clerked all acute admissions during the day. In addition, they assisted the FY1 attached to the admitting consultant when necessary. This allowed the workload of acute surgical patients to be managed more fairly, while still ensuring that emergency admissions were clerked by an FY1.

\section{PDSA cycle 3}

Although more admissions were being clerked by FY1 doctors, many were still missed. The underlying factor was felt to be uncertainty amongst nursing staff about which FY1 to contact when a new admission reached the ward. A baton bleep was created which was to be carried by the FY1 responsible for emergency admissions. All surgical ward managers and nursing staff were informed of the new bleep number and it was added to the existing FY1 bleep lists on noticeboards on the surgical wards.

\section{Results}

The same online survey was administered two months after the baseline survey. At this point, the baton bleep and dedicated emergency FY1 system had been in place for a one month period. A $100 \%(n=10)$ return rate for questionnaires was achieved. In this short time the results showed a significant improvement.

FY1s feeling that exposure to surgical emergencies was adequate improved from $0 \%$ to $100 \%(n=10)$. This was associated with an increase in the reported average number of acute admissions seen per week from 1.2 to 4.0 patients (figure 1).

Increasing the number of opportunities to present cases and discuss them with seniors had been a clear goal, and where only $60 \%(n=6)$ of respondents had initially felt that they were able to do this either frequently or sometimes, this rose to $90 \%(n=9)$ after intervention.

Confidence in diagnosis of surgical emergencies rose from $0 \%$ to $40 \%(n=4)$, while confidence in initial management and investigation rose from $60 \%(n=6)$ to $100 \%(n=10)$ (figure 2$)$.

The most concerning result from the baseline survey was that only $30 \%$ of $\mathrm{FY} 1$ doctors felt that their current job was providing them with the relevant skills and experience to become an $\mathrm{SHO}$ in general surgery. Following the intervention, with increased exposure to emergency cases, $100 \%(n=10)$ felt that they were being prepared for the next stage.

See supplementary file: ds2980.docx - "Results" 


\section{BMJ Quality Improvement Reports}

\section{Lessons and limitations}

The most junior members of our team can easily be sidelined due to the fragmentation of the traditional surgical firm. When they are only required to complete routine ward based tasks, they can become disillusioned, feel undervalued, and miss out on valuable learning opportunities.

This project has emphasised the importance of acute admissions to the training of junior doctors. By including the FY1 in the admission and subsequent patient journey, you increase experience and confidence. The knock on effect of this is to provide better assessment and management of patients as these doctors progress to FY2 level.

The most significant improvement brought about through these interventions has been that prior to increasing exposure to acute admissions, only $30 \%$ of the $\mathrm{FY} 1$ doctors felt that they were being adequately prepared for a job as an FY2 in general surgery, with this increasing to $100 \%$ following intervention. The benefits of this will certainly extend beyond the realms of general surgery, as many of the skills learned will be transferrable to other specialities.

Following the changes, the FY1s reported feeling more involved as part of the surgical team, a further benefit from the changes to working practice.

Although significant progress was observed during the study period, there were times when staffing levels were reduced and FY1 involvement in emergency cases fell. Enthusiasm and support from more senior members of the admitting team is necessary to maintain FY1 involvement and to ensure that their hard work is rewarded with opportunities to learn and develop clinical skills.

\section{Conclusion}

Changes to modern working practices should not be at the expense of the education and development of junior doctors. Emergency admissions must remain an integral part of the role of FY1 doctors. We have demonstrated that by making adjustment to the working structure of junior doctors, exposure to emergencies can be promoted, and the knock on effect is more experienced and more confident trainees.

Earlier exposure to emergencies prepares FY1s for more senior roles.

\section{References}

1. Gossage JA, Modarai B, McGuinness CL, Burnand KG., The modernisation of the surgical house officer, Ann R Coll Surg Engl. 2005 Sep;87(5):369-72.

2. The UK Foundation Program Curriculum, July 2012.

3. National Training Survey 2013 report- Foundation Training.

4. Rachel T Clarke, Alex Pitcher, Trevor W Lambert, Michael J Goldacre, UK doctors' views on the implementation of the European Working Time Directive as applied to medical practice: a qualitative analysis, BMJ Open. 2014; 4(2): e004390.

\section{Declaration of interests}

None

\section{Acknowledgements}

None 